\title{
Un Controlador Basado en la Fusión de Controladores PVTOL para Navegación 3D de Vehículos Aéreos Autónomos
}

\section{Milton Cesar P. Santos}

Instituto Federal do Espirito Santo, Rod ES-080, km 93, 29.660-000 Santa Teresa, ES, BRASIL

miltoncps@gmail.com

\section{Mario Sarcinelli-Filhot}

Universidade Federal do Espirito Santo, Av. Fernando Ferrari, 514, 29.075-910 Vitoria, ES, BRASIL mario.sarcinelli@ufes.br

\section{Alexandre S. Brandaot}

Universidade Federal de Vicosa, Av. P. H. Rolfs, S/N, 36.570-000 Vicosa, MG, BRASIL

alexandre.brandao@ufv.br

\section{Ricardo Carelli}

Universidad Nacional de San Juan, Av San Martin 1109, Oeste, 5400ARL San Juan, ARGENTINA

rcarelli@inaut.unsj.edu.ar 


\title{
Resumen
}

En este trabajo se propone una arquitectura de control basada en la fusión de las acciones de control generadas por tres controladores sencillos (controladores PVTOL, del inglés Planar Vertical Take-Off and Landing) para guiar vehículos aéreos autónomos en posicionamiento y seguimiento de caminos 3D. Considerando la navegación como una tarea compuesta por el control de la orientación del vehículo en relación al blanco (asociada a un controlador Z-PVTOL), por el control de su movimiento hacia adelante (asociado a un controlador XZ-PVTOL), y por la corrección de cualquier desplazamiento lateral (asociada a un controlador YZ-PVTOL, similar al controlador XZ-PVTOL), nuestro primer abordaje consistió en ejecutar dichas maniobras parciales una por vez, utilizando los controladores PVTOL, conmutando de uno al otro mediante la acción de un supervisor. En este trabajo el abordaje se cambió, pasando a considerarse que los controladores PVTOL actúan simultáneamente y generando la acción de control final mediante la fusión de las acciones de control generadas por ellos, acoplando así suavemente tres controladores muy sencillos. Se presentan resultados experimentales para validar el abordaje propuesta, y su desempeño en vuelo es comparado con el desempeño correspondiente a la estrategia de conmutación.

Palabras clave: Fusión de acciones de control, VANT, Navegación autónoma, Robótica aérea

\begin{abstract}
This paper proposes a control architecture based on the fusion of control actions generated by three simple controllers (PVTOL controllers, of the English Planar Vertical TakeOff and Landing) to guide autonomous aerial vehicles in 3D path positioning and tracking. Considering navigation as a task composed of the control of the orientation of the vehicle in relation to the target (associated with a Z-PVTOL controller), the control of its forward movement (associated with an XZ-PVTOL controller), and the Correction of any lateral displacement (associated with a YZ-PVTOL controller, similar to the XZ-PVTOL controller), our first approach consisted in executing said partial maneuvers one at a time, using the PVTOL controllers, switching from one to the other through the action of a supervisor. In this work the approach was changed, considering that the PVTOL controllers act simultaneously and generating the final control action by merging the control actions generated by them, thus gently coupling three very simple controllers. Experimental results are presented to validate the proposed approach, and its flight performance is compared with the performance corresponding to the switching strategy.
\end{abstract}

Keywords: Fusion of control actions, VANT, Autonomous navigation, Aerial robotics

\section{INTRODUCCIÓN}

En los últimos años es notable el gran interés de la comu-nidad científica por vehículos aéreos no tripulados (VANT), en particular por su navegación autónoma. Esto es debido principalmente a algunas características de tales aeronaves que facilitan la ejecución de 
ciertas tareas, en una gran diversidad de áreas, tales como seguridad pública (por ejemplo supervisión del espacio aéreo y del tráfico urbano), gestión de riesgos naturales (por ejemplo vigilancia de volcanes activos), gestión ambiental (por ejemplo medición de polución del aire y supervisión de florestas), intervenciones en ambientes hostiles (por ejemplo atmosferas radiactivas), mantenimiento de infraestructuras (por ejemplo inspección de líneas de transmisión y tubería de agua u óleo), y agricultura de precisión (por ejemplo detección y tratamiento de plantaciones infestadas), además de otras áreas. En dichas aplicaciones el uso de VANTs es extremadamente ventajoso comparado con el uso de vehículos terrestres no tripulados (VTNTs), debido a su movilidad tridimensional [1] - [4].

Dichas aplicaciones requieren las capacidades de volar en baja altitud, permanecer parado y despegar y aterrizar verticalmente (VTOL, del inglés Vertical Take-Off and Landing), de suerte que pequeñas aeronaves autónomas de alas rotativas (RUAS, del inglés Rotorcraft Unmanned Aircraft Systems) son más apropiadas para tales aplicaciones que aeronaves autónomas de alas fijas [5]. Esto porque un factor crucial del helicóptero y sus vehículos semejantes, como el cuatrimotor, es la posibilidad de control en las direcciones lateral, longitudinal y vertical, con cualquier orientación. Dicha característica confiere una capacidad superior de maniobras a los vehículos de alas rotativas, lo que es un factor que los distingue de las aeronaves de alas fijas [6]. Una desventaja, sin embargo, es el mayor consumo de energía durante el vuelo, lo que resulta que en general vuelos con pequeños helicópteros o similares son de corta duración.

Tratándose específicamente de las familias de aeronaves de alas rotativas, un cuatrimotor es mucho más simple y fácil de construir, comparado al helicóptero clásico, debido a que no incluye el conjunto del plato oscilante (del termino en ingles swashplate). Más aun, él es controlado solamente variándose la rotación de cada uno de sus cuatro motores, los cuales giran en sentido contrario dos a dos, lo que hace dichas aeronaves muy atractivas como plataformas para ensayar estrategias de control. En este trabajo en particular, el cuatrimotor AR.Drone 2.0, de Parrot Inc., ilustrado en la Figura I, es la plataforma adoptada para realizar los experimentos descritos más adelante.

El control de los RUAS ha atraído la atención de muchos investigadores, tanto de la comunidad de control cuanto de la comunidad de robótica, debido a los desafíos y a la oportu nidad de desarrollar y ensayar nuevas estrategias de control. De hecho, una gran variedad de artículos sobre el control de aeronaves de alas rotativas ya está disponible en la literatura. Algunos de ellos son basados en control adaptable, como en [7], donde se propone un controlador no lineal adaptable para vuelo basado en visión utilizando un cuatrimotor. Otros trabajos usan la técnica de backstepping, como en [8]. A su vez, en [9] el algoritmo desarrollado genera trayectorias óptimas por medio de una secuencia de posiciones 3D y ángulos de guiñada. Tales trayectorias son entonces seguidas con precisión, usando un controlador no lineal con lazos interno y externo (del inglés inner- and outer-loop controller). En el caso de [10] se propone un esquema de control no lineal en tiempo real con saturación, basado en el criterio de estabilidad de Lyapunov. Otros ejemplos de estrategias de control se encuentran en [11] y en [12], donde se propone un controlador sub-actuado. También se puede citar [13], donde los autores comparan el desempeño de su controlador no lineal con el de un controlador lineal, en este caso el regulador lineal cuadrático (LQR, del inglés Linear Quadratic Regulator), lo cual presenta problemas de estabilidad cuando el sistema opera lejos del punto de operación utilizado en el proyecto del controlador lineal. 


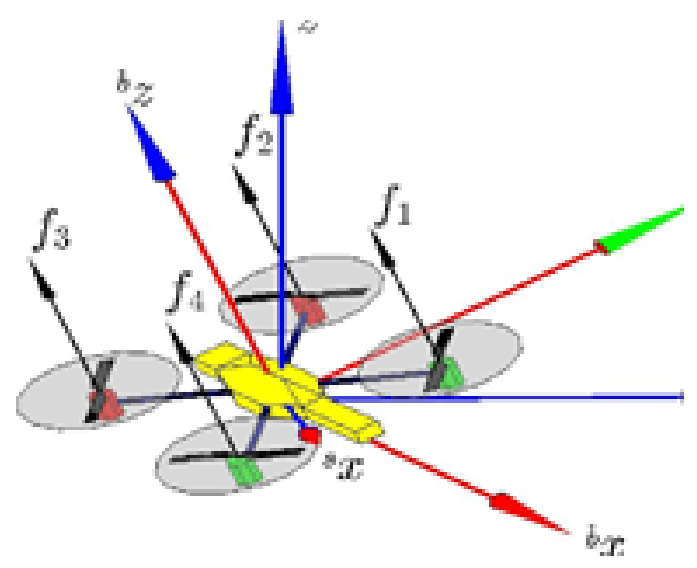

Figura 1. Modelo de 6 GDL del cuatrimotor AR.drone, mostrando los sistemas de referencia asociados a 61 y sus entradas de control abstractas fi, $i=1,-, 4$. Los sistemas inercial, espacial y del cuerpo del vehículo son (g), (s) y (b), como está indicado por el índice a la izquierda en los ejes x, y y z.

Nótese que todos los trabajos citados arriba, así como muchos otros, se basan en el modelo no lineal del vehículo, lo que muchas veces hace difícil implementar las leyes de control, debido a la gran demanda computacional del algoritmo o bien por el hecho de que el grado de idealismo del modelo es tal que es imposible realizar un experimento fuera del laboratorio. Una alternativa es presentada en [14], donde los autores introducen el concepto de tareas PVTOL, que corresponden a vuelos restringidos a un plano vertical. 0 sea, la aeronave despega verticalmente, se orienta en relación al blanco, avanza en línea recta hasta alcanzarlo, para y aterriza. Tal tipo de tarea es un benchmark importante para proyectos de control, teniendo como objetivo estabilizar y mover una aeronave de alas rotativas en un plano vertical. Respecto a las aeronaves de alas rotativas convencionales, esto se puede lograr restringiendo algunos grados de libertad del vehículo, de modo que de acuerdo a los grados de libertad restringidos diferentes tareas PVTOL son cumplidas. Si la tarea se realiza en el plano XZ, por ejemplo, los movimientos de guiñada y rolido deben ser restringidos (vea [15]). Cuando la tarea debe ser cumplida en el plano YZ, los movimientos de guiñada y cabeceo son los que deben ser restringidos, mientras que una tarea VTOL (vuelo parado) exige que los desplazamientos longitudinal y lateral sean restringidos. Tales tareas PVTOL son ilustradas en la Figura I. 


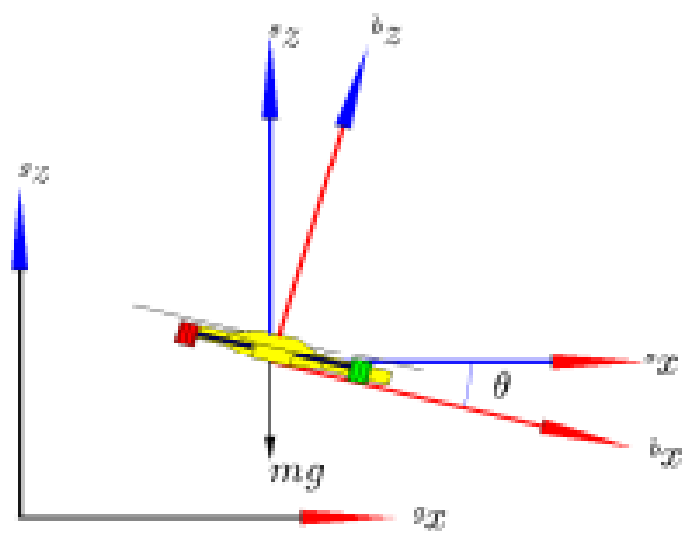

(a) Caracterización de la tarea PVTOL en el plano $X Z$.

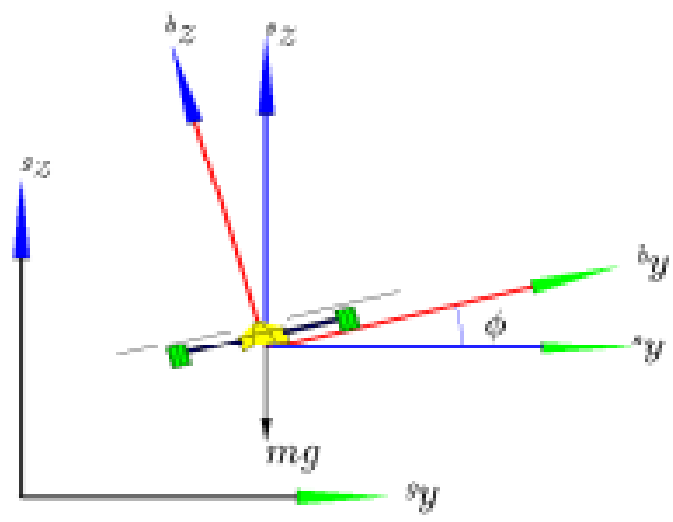

Figura 2. Caracterización de los movimientos PVTOL para el cuatrimotor AR.Drone, de Parrot, Inc.

Un aspecto importante de las tareas PVTOL es que ninguna limitación es impuesta a su punto de operación, a diferencia de lo que ocurre en los casos de linealización. Es decir, aun para tareas PVTOL controladores no lineales tienen desempeño superior a los casos de controladores lineales, basados en modelos linealizados.

Este trabajo trata del control de una aeronave de alas rotativas combinando diferentes controladores PVTOL para posicionar un cuatrimotor en el espacio 3D, en que cada uno de los controladores está basado en la teoría de Lyapunov. Más aun, se puede especificar una secuencia de puntos para que el cuatrimotor alcance cada uno de ellos, lo que corresponde a una tarea de seguimiento de camino. Por lo tanto, el con-trolador propuesto es, en principio, compatible con tareas de posicionamiento y seguimiento de caminos en el espacio 3D. Se debe notar que la combinación de controladores PVTOL que acá se propone es diferente de aquella ya propuesta en [16]: allí los controladores PVTOL se combinan por medio de 
conmutación entre ellos, mientras que en este trabajo dicha combinación ocurre por medio de la fusión de las acciones de control generadas por cada uno de los tres controladores PVTOL. La razón por la cual se desarrolló esta metodología es que la conmutación entre los controladores PVTOL hace con que el cumplimiento de una tarea de posicionamiento tome mucho tiempo, lo que en la práctica imposibilita utilizarse tal esquema en tareas de seguimiento de trayectorias, por ejemplo, en que los requisitos de velocidad son impuestos por la trayectoria que está siendo seguida. La expectativa era que la navegación basada en la fusión de las acciones de control de los controladores PVTOL sería mucho más rápida, lo que de hecho ocurre, conforme se verá en la secuencia del texto.

Para abordar tales tópicos, este artículo se divide, desde este punto, en las siguientes secciones: Sección II, que describe de manera bastante sucinta los tres controladores PVTOL utilizados, Sección III, que describe el esquema de fusión utilizado para combinar las acciones de control provistas por los tres controladores PVTOL utilizados para generar la acción de control final aplicada al cuatrimotor, Sección IV, que trata de algunos experimentos realizados, y, por fin, Sección V, en que se listan las principales conclusiones del trabajo.

\section{CONTROLADORES PVTOL UTILIZADOS}

Como ya se mencionó, tres controladores proyectados para guiar el VANT en tareas PVTOL son implementados, y las acciones de control por ellos generadas son insertadas en un filtro de información descentralizado [17] - [19], utilizado como máquina para fusión de datos. Así será generada una sola acción de control, la cual será enviada al cuatrimotor para guiarlo desde su posición actual hasta una posición deseada, cumpliendo así una tarea de posicionamiento en el espacio 3D. La esencia es que controladores para movimiento bidimensional (PVTOL) son utilizados para guiar la navegación en espacio tridimensional, aprovechando el hecho de que tales controladores son más sencillos de diseñar e implementar.

El primero de tales controladores es un controlador para el movimiento de ascenso/descenso, es decir, desplazamiento lineal en la dirección del eje $\mathrm{z}$, y rotación a su alrededor. Para esto los ángulos de cabeceo y rolido son restringidos a permanecer nulos durante toda la navegación. En cuanto al segundo de tales controladores, él tiene por objetivo permitir ascender y descender el cuatrimotor y moverlo hacia adelante o hacia eras. Es decir, ahora los ángulos de cabeceo y guiñada son restringidos a permanecer en cero durante toda la navegación. Por fin, en el caso del tercer controlador el movimiento deseado es similar al del segundo caso, con la diferencia que ahora los ángulos restringidos a permanecer en cero son los ángulos de cabeceo y guiñada. Así se tiene un movimiento PVTOL solamente en la dirección del eje z y con giro a su alrededor, un movimiento en la dirección del plano XZ y un movimiento en la dirección del plano YZ, siendo estos dos últimos caracterizados en la Figura I. Los controladores correspondientes a cada uno de dichos movimientos son discutidos en detalle en [16] y [15], y por consiguiente no serán detallados acá.

Por fin, vale mencionar que la propuesta de utilizar un controlador basado en la conmutación entre los tres controladores PVTOL mencionados, conforme a lo discutido en [16], también se implementa en este trabajo, a efectos de comparar los resultados de ambos abordajes. 


\section{FUSIÓN DE LOS CONTROLADORES PVTOL}

Para realizar la fusión de datos se pueden utilizar dos métodos, que son el filtro de Kalman [20], [21] y el filtro de información [22]. Dichos filtros permiten fusionar datos provistos por múltiples sensores/sistemas, resultando en datos de salida con varianza menor que la menor varianza asociada a los datos de entrada [17].

El filtro de información es esencialmente un filtro de Kalman con las ecuaciones escritas en función de la información sobre medidas de los estados de interés [18], y no de las estimas de dichos estados y las covarianzas a ellas asociadas [17]. En este trabajo, fue utilizado el filtro de información, en su versión descentralizada, como máquina para la fusión de datos, por presentar la ventaja, frente al filtro de Kalman descentralizado, de que sus ecuaciones son más sencillas de implementar y ser más robusto en términos de su inicialización. De hecho, para inicializar el filtro de información descentralizado basta considerar un vector de información de los estados (y) igual a cero y una matriz de información (Y) próxima de cero, aunque no nula, mientras que en el caso del filtro de Kalman es necesario un conocimiento previo del sistema para tener una buena estima inicial del vector de estados y de la matriz de covarianza del error.

\section{Filtro de información}

Las ecuaciones del filtro de información se basan en

$$
Y_{(k)}=P_{(k \mid k-1)}^{-1}
$$

y

$$
\hat{y}_{(k)}=P_{(k \mid k-1)}^{-1} \hat{x}_{(k \mid k-1)}=Y_{(k)} \hat{x}_{(k \mid k-1)},
$$

que corresponden, respectivamente, a la matriz de información y al vector de información de los estados.

Adoptándose dichos valores, las ecuaciones correspondientes al filtro propiamente son son, respectivamente, el coeficiente de propagación de la información, la contribución de la información de los estados y la matriz de información, todos asociados a cada estado.

Predicción:

$$
\begin{aligned}
\hat{y}_{(k \mid k-1)} & =L_{(k)} \hat{y}_{(k-1 \mid k-1)} \\
Y_{(k \mid k-1)} & =\left[A_{(k)} Y_{(k-1 Z k-1)}^{-1} A_{(k)}^{T}+Q_{(k)}\right]^{-1}
\end{aligned}
$$




\section{Estimación:}

$$
\begin{aligned}
& \hat{y}_{(k \mid k)}=\hat{y}_{(k \mid k-1)}+i_{(k)} \\
& Y_{(k \mid k)}=Y_{(k \mid k-1)}+I_{(k)},
\end{aligned}
$$

donde

$$
\begin{aligned}
L_{(k)} & =Y_{(k \mid k)} A(k) Y_{(k \mid k-1)}^{-1} \\
i_{(k)} & =H_{(k)}^{t} R_{(k)}^{-1} z_{(k)} \\
I_{(k)} & =H_{(k)}^{t} R_{(k)}^{-1} H_{(k)}
\end{aligned}
$$

\section{Filtro de información descentralizado}

Como el filtro de información es una derivación del filtro de Kalman, la formulación del filtro de información descentralizado es similar a la del filtro de Kalman descentralizado. Así, para el i-ésimo filtro local se tiene

$$
\begin{aligned}
& \hat{y}_{i(k)}=\hat{y}_{(k \mid k-1)}+i_{i(k)} \\
& Y_{i(k)}=Y_{(k \mid k-1)}+I_{i(k)},
\end{aligned}
$$

mientras que para el filtro global se tiene

$$
\begin{aligned}
& \hat{y}_{(k)}=\sum_{i}^{n} \hat{y}_{i(k)}-(n-1) \hat{y}_{(k \mid k-1)} \\
& Y_{(k)}=\sum_{i}^{n} Y_{i(k)}-(n-1) Y_{(k \mid k-1)} .
\end{aligned}
$$

La Figura 3 ilustra el diagrama del filtro de información descentralizado con i filtros de información locales.

\section{La arquitectura de control propuesta}

La arquitectura de control acá propuesta corresponde a un filtro de información descentralizado con tres filtros locales, que reciben las acciones de control generadas por los tres controladores PVTOL que se mencionaron en la Sección II. Dicha arquitectura está en la Figura 4, donde se puede apreciar que cada uno de los tres controladores PVTOL recibe información del VANT y calcula la acción de control correspondiente, que pasa a los filtros de información locales, que a su vez entregan la información al filtro de información global, lo que corresponde a un método de fusión recursivo y optimizado [17], [18]. 
Lo más importante, considerando la estrategia propuesta, es como seleccionar el peso correspondiente a cada filtro local para guiar el VANT en su tarea de posicionamiento o seguimiento de camino. En resumen, la arquitectura de control propuesta determina la matriz de covarianza del error en las observaciones (acciones de control provistas por cada uno de los controladores XZ-PVTOL, YZ-PVTOL y Z-PVTOL) con pesos calculados a partir de los errores de posición y velocidad del VANT. En principio, el método propuesto es una evolución de la arquitectura de controladores conmutados: en lugar de la conmutación entre ellos, se utiliza una fracción de cada controlador, de acuerdo con su covarianza asociada.

\section{Estimando las acciones de control para el VANT}

La arquitectura propuesta tiene como variables de salida para cada controlador $i$

$$
\hat{\mathbf{x}}_{i}=\left[\begin{array}{llll}
u_{\theta} & u_{\phi} & u_{\dot{\psi}} & u_{\dot{z}}
\end{array}\right],
$$

donde uz ${ }^{\bullet}$ representa un comando de velocidad linear que causadesplazamiento en el eje $\mathrm{zW}, \mathrm{u} \psi^{\bullet}$ representa un comando develocidad angular que causa la rotación al rededor del eje zw, uф representa, indirectamente, un comando de velocidad lineal que causa desplazamiento en el eje yb (indirectamente porque por medio del ángulo de rolido $\phi$ ), y, por fin, $\mathrm{u} \theta$ representa, también indirectamente, por medio del ángulo de cabeceo $\theta$, un comando de velocidad lineal que causa desplazamiento en el eje $\mathrm{xb}$.

Como se ha dicho anteriormente, la información de entrada para estimar las acciones de control para el VANT vienen de los controladores XZ-PVTOL ( $\mathrm{i}=1)$, YZ-PVTOL $(\mathrm{i}=2) \mathrm{y}$ Z-PVTOL ( $\mathrm{i}=3$ ). Así, el filtro global tiene tres filtros locales, y para cada filtro local i la configuración general del vector de medidas, modelo de observación y matrices de covarianza del error de medición corresponden a 


$$
\begin{aligned}
& \mathbf{z}_{i}=\left[\begin{array}{llll}
u_{\theta} & u_{\phi} & u_{\dot{\psi}} & u_{i}
\end{array}\right], \\
& H_{i}=\left[\begin{array}{llll}
1 & 0 & 0 & 0 \\
0 & 1 & 0 & 0 \\
0 & 0 & 1 & 0 \\
0 & 0 & 0 & 1
\end{array}\right], \quad \mathrm{y} \\
& R_{i}=\left[\begin{array}{cccc}
\sigma_{i x}^{2} & 0 & 0 & 0 \\
0 & \sigma_{i y}^{2} & 0 & 0 \\
0 & 0 & \sigma_{i v}^{2} & 0 \\
0 & 0 & 0 & \sigma_{i s}^{2}
\end{array}\right] .
\end{aligned}
$$

Considerando las particularidades de cada controlador, se tienen las configuraciones

- Filtro-1:

$$
\begin{aligned}
& \mathbf{z}_{1}=[u_{\theta} \overbrace{u_{\phi}}^{0} \overbrace{u_{\psi}}^{0} u_{t}], \quad \mathbf{y} \\
& R_{1}=\left[\begin{array}{cccc}
\frac{1}{\sigma(\hat{x}, \hat{x})} & 0 & 0 & 0 \\
0 & \sigma(\tilde{y}, \hat{y}) & 0 & 0 \\
0 & 0 & \sigma(\tilde{\psi}, \hat{\psi}) & 0 \\
0 & 0 & 0 & \frac{1}{\sigma(I, \dot{H})}
\end{array}\right] ;
\end{aligned}
$$

- Filtro-2:

$$
\begin{aligned}
& \mathbf{z}_{2}=[\overbrace{u_{\theta}}^{0} u_{\phi} \overbrace{u_{\dot{\psi}}}^{0} u_{i}], \quad \mathrm{y} \\
& R_{2}=\left[\begin{array}{cccc}
\sigma(\tilde{x}, \dot{\hat{x}}) & 0 & 0 & 0 \\
0 & \frac{1}{\sigma(\underline{y},)} & 0 & 0 \\
0 & 0 & \sigma(\dot{\psi}, \hat{\psi}) & 0 \\
0 & 0 & 0 & \frac{1}{\sigma(I, T)}
\end{array}\right] ;
\end{aligned}
$$




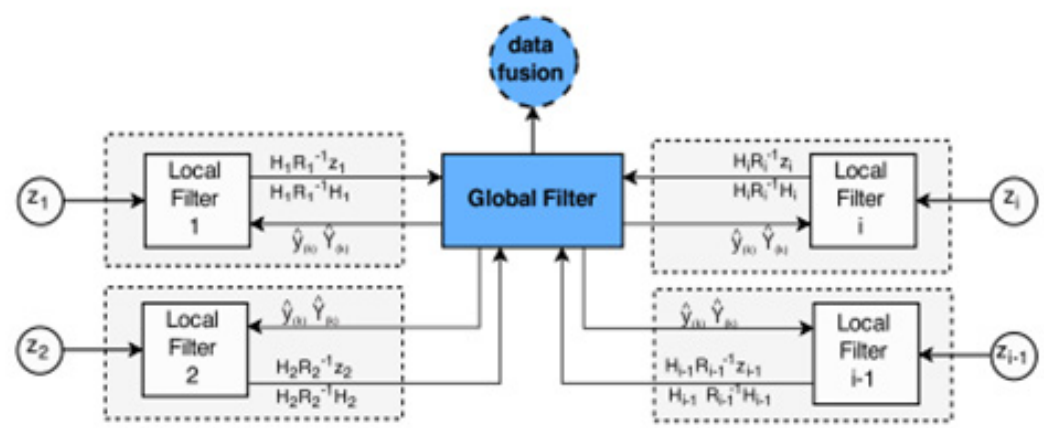

Figura 3. Diagrama del filtro de información descentralizado con $i$ filtros de información locales, donde $H, R$ y z son el modelo de obscrvación, la estima de la covarianza del ruido y el vector de mediciones, respectivamente.

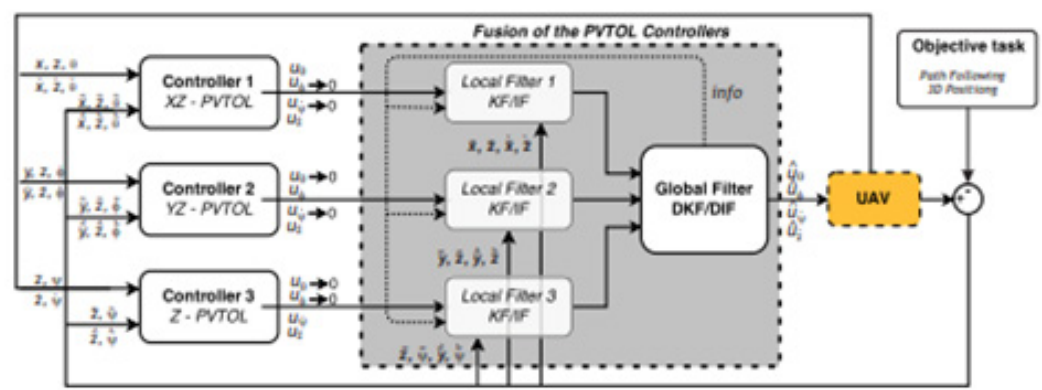

Figura 4. Arquihectura de control propuesta. En el bloque fasión la anformacin exhibida corresponde a $y_{(a)}, \hat{y}_{(\alpha)}$.

- Filtro-3:

$$
\begin{aligned}
& \mathbf{z}_{3}=\overbrace{u_{\theta}}^{0} \overbrace{u_{\phi}}^{0} u_{\dot{\psi}} u_{\dot{i}}], \quad \mathrm{y} \\
& R_{3}=\left[\begin{array}{cccc}
\sigma(\tilde{x}, \hat{x}) & 0 & 0 & 0 \\
0 & \sigma(\tilde{y}, \hat{y}) & 0 & 0 \\
0 & 0 & \frac{1}{\sigma(\hat{\varphi}, \hat{\varphi})} & 0 \\
0 & 0 & 0 & \frac{1}{\sigma(\tilde{y}, \dot{y})}
\end{array}\right] \text {, } \\
& \text { en que } \sigma(\tilde{x}, \dot{\hat{x}})=\sqrt{\rho_{x}+\kappa_{x}\left(\hat{x}^{2}+\hat{x}^{2}\right)}, \sigma(\hat{y}, \dot{y})=\sqrt{\rho_{y}+\kappa_{y}\left(\hat{y}^{2}+\dot{y}^{2}\right)} \text {, } \\
& \sigma(\tilde{\psi}, \dot{\psi})=\sqrt{\rho_{\psi}+\kappa_{x}\left(\tilde{\psi}^{2}+\dot{\psi}^{2}\right)} \text { y } \sigma(z, \tilde{z})=\sqrt{\rho_{z}+\kappa_{z}\left(z^{2}+\dot{z}^{2}\right)} \text { son } \\
& \text { funciones que definen el peso relativo instantáneo de cada controla- } \\
& \text { dor. }
\end{aligned}
$$

dor.

El cálculo de las covarianzas relacionadas con las acciones de control se hace obedeciendo una lógica de relevancia. Por ejemplo, en el filtro-1 el controlador XZ-PVTOL calcula solamente las acciones[u $\left.\mathrm{uz}^{*}\right]$, luego el peso referente a dichas acciones debe ser mássignificativo. Las funciones $\sigma\left(\mathrm{x}^{\sim}, \mathrm{x}^{\sim}\right), \sigma\left(\mathrm{y}^{\sim}, \mathrm{y}^{\sim}\right), \sigma\left(\psi^{\sim}, \psi^{\sim}\right)$ y $\sigma\left(\mathrm{z}^{\sim}, \mathrm{z}^{\sim}\right)$ generan valores positivos 
que influencian en la estima e indican la significancia de los datos observados. En este caso valores para covarianzapróximos a cero indican que las observaciones son más relevantes.

De esta forma se observa que para el filtro-1, que actúa efectivamentecon [u$\theta \mathrm{uz}{ }^{*}$ ], la función de peso es $\sigma\left(\mathrm{x}^{\sim}, \mathrm{x}^{\sim}\right)-1$ y $\sigma\left(\mathrm{z}^{\sim}, \mathrm{z}^{\sim}\right)-1$ com alta pertinencia, al paso que las demás acciones de control, [u $\phi \mathrm{u} \psi^{*}$ ] son sometidas a pesos con baja pertinencia $\sigma\left(\psi^{\sim}, \psi^{\sim}\right)$ y $\sigma\left(\mathrm{z}^{\sim}, \mathrm{z}^{\sim}\right)$.

Así, los pesos relativos asociados a las acciones de control seránfunción de cuán .observables"son en las mediciones. Acciones inmediatamente visibles en las mediciones reciben pesos más grandes.

Una vez que el filtro calcula una estima actualizada de los estados usando las nuevas mediciones, la covarianza de los estados estimados también debe ser modificada, para reflejar la información añadida, disminuyéndose así la incertidumbre. Así, dichos filtros fusionan las acciones de control por los tres controladores, resultando en una acción de control cuya varianza es más pequeña que la más pequeña varianza asociada a las acciones de control generadas por cada controlador individualmente. La salida del filtro global, entonces, es

$$
\hat{\mathbf{x}}_{\text {fusion }}=\left[\begin{array}{llll}
\hat{u}_{\theta} & \hat{u}_{\phi} & \hat{u}_{\dot{\psi}} & \hat{u}_{\dot{t}}
\end{array}\right] \text {. }
$$

Por fin, se comenta que hasta ahora no se ha obtenido una prueba formal de la estabilidad del sistema de control propuesto. Sin embargo, conjeturamos que de hecho el sistema es estable, conjetura que se apoya en el hecho de que no hay discontinuidad en la acción de control aplicada al VANT, como es el caso cuando ocurre la conmutación entre controladores (la acción de control acá generada tiene variación suave a lo largo del vuelo), y en los resultados experimentales obtenidos utilizándose la arquitectura propuesta, descritos a continuación.

\section{RESULTADOS EXPERIMENTALES}

Dos experimentos fueron realizados con el cuatrimotor AR.Drone,de Parrot, Inc., para comparar el desempeño de las estrategias decontrol basadas en la conmutación entre controladores PVTOL y enla fusión de las acciones de control generadas por ellos. Se trata de dos tareas de seguimiento de camino, uno en forma de ocho y otroen espiral, ambos en el espacio tridimensional.

Las dos tablas a continuación permiten comparar las dos estrategias de control. La Tabla I se refiere al primer experimento (camino 61 en forma de ocho), mientras que la Tabla II se refiere al segundoexperimento (camino espiral). Dichas tablas muestran el tiempo derecorrido y los índices de desempeño ISE (integral del cuadrado delerror) e IAE (integral del valor absoluto del error), para el caso deconmutación entre los controladores y para el caso de fusión de las acciones de control que acá se propone. 
TABLA I

EXPERIMENTO 1: SEGUIMIENTO DE CAMINO EN FORMA DE OCHO

\begin{tabular}{lccc}
\hline Estrategia & Tiempo [s] & ISE & IAE \\
\hline Conmutación & 448.0740 & 9.0940 & 80.9668 \\
Fusi6n & 124.6410 & 3.8203 & 21.5379 \\
\hline
\end{tabular}

TABLA II

EXPERIMENTO 2: SEGUIMIENTO DE CAMINO EN ESPIRAL

\begin{tabular}{lccc}
\hline Estrategia & Tiempo [s] & ISE & IAE \\
\hline Conmutación & 690.95 & 44.2309 & 188.0470 \\
Fusi6n & 214.83 & 22.4165 & 61.2083 \\
\hline
\end{tabular}

Además de dichas tablas, las Figuras 5 y 6 a continuación traen las gráficas correspondientes a los mismos experimentos.

Un análisis de los resultados experimentales comprueba que la estrategia de fusión de las acciones de control obtenidas de los controladores PVTOL para generar un controlador capaz de guiar el VANT en tareas de navegación 3D tiene dos ventajas bastante destacadas: la primera es que la misma tarea es ejecutada en mucho menos tiempo, comparada con la estrategia de conmutación entre los mismos controladores, y la segunda es que los errores de posicionamiento son mucho más pequeños, además del hecho que el camino seguido es más suave. Incluso, se debe destacar que el menor tiempo de ejecución de la tarea hace viable la utilización del sistema de control propuesto en tareas de seguimiento de trayectoria, lo que

no es posible en el caso de conmutación entre los controladores, como se destaca en [16].

\section{Conclusiones}

En este trabajo se propone una arquitectura de control basada enla fusión de las acciones de control generadas por tres controladoresrestringidos para ejecución de tareas PVTOL con un cuatrimotor, del que resulta una navegación en el espacio 3D. Aunque el objetivo de navegar en el espacio 3D usando controladores de alto nivel correspondientes a navegación $1 \mathrm{D}$ y $2 \mathrm{D}$ ya ha sido explorada a través de un esquema de conmutación entre los controladores, la solución por medio de la fusión de las acciones de control individuales acá propuesta es innovadora, y se ha mostrado bastante más efectiva que la conmutación entre dichas acciones de control, resultando en una navegación más rápida y más suave, en los casos de posicionamiento y seguimiento de caminos. También se propone una forma analítica de asignar el peso correspondiente a cada uno de los tres controladores básicos en función de 
los errores con respecto al posicionamiento deseado, función esta que es actualizada a cada ciclo de control.

De este modo lo que se obtiene es una ley de control continua y única, expresada por medio de una función analítica, la cual, intrínsecamente, permite dar más peso al controlador básico que más se adapte a la tarea en cada ciclo de control (aún considerando también las contribuciones de los otros dos). Mas aún, también se muestra que la fusión de las diferentes acciones de control, generadas por controladores que poseen distintos objetivos de control, pero comparten fracciones del objetivo común, genera una acción de control acotadas cuando se aplican las funciones de peso propuestas.

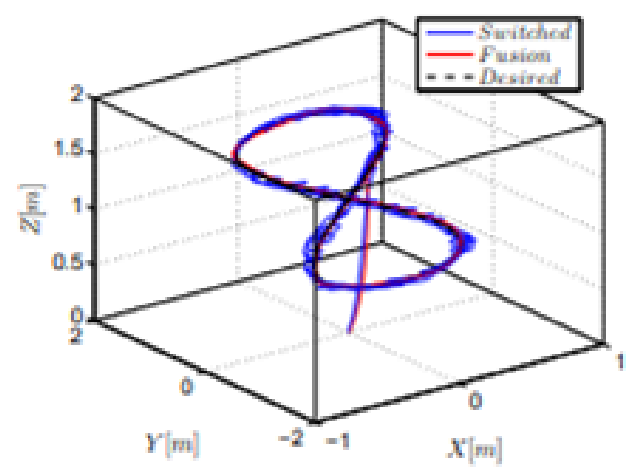

(a)

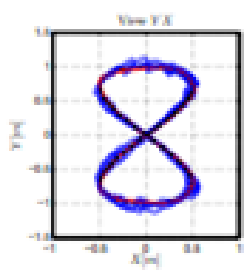

(b)

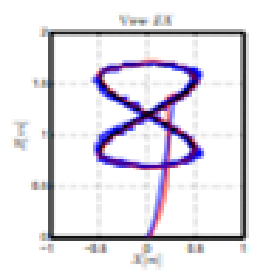

(c)

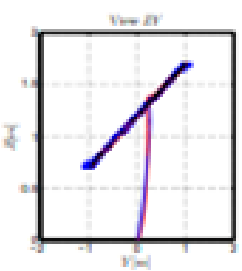

(d) 


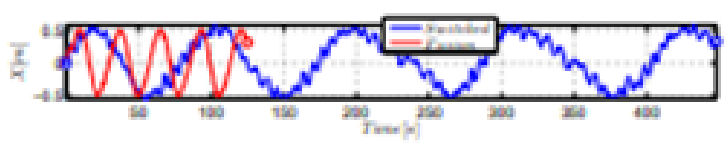

(e)

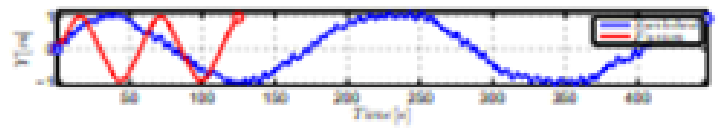

(f)

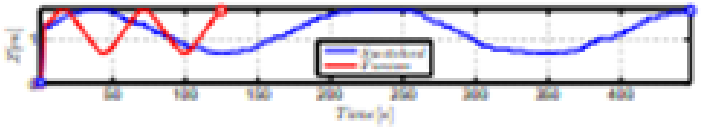

(g)



(h)

Figura 5. Grifieas eorrespondientes al experimento 1.

Finalmente, aunque la estabilidad del sistema de control como un todo no ha sido probada, se propuso la conjetura de que el sistema de control en lazo cerrado, cuando el controlador basado en la fusión de las acciones de control es utilizado, es estable, siendo dicha conjetura basada en el hecho de que la acción de control no sufre discontinuidad, como ocurre en la conmutación entre controladores, y en los resultados experimentales presentados.

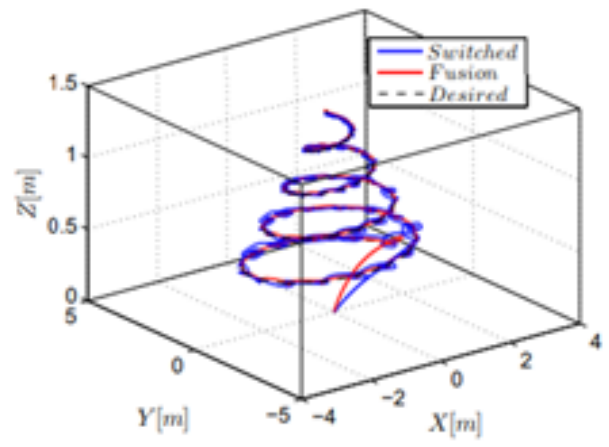

(a)

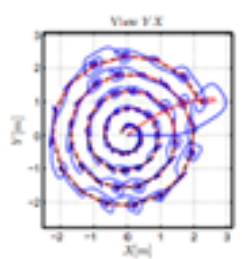

(b)

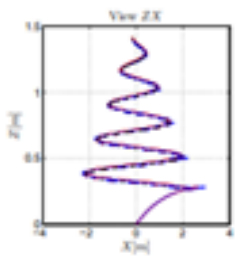

(c)

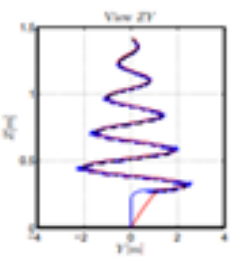

(d) 


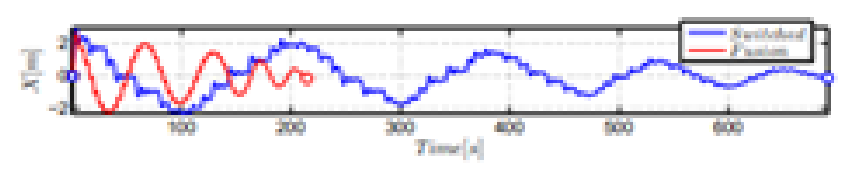

(e)

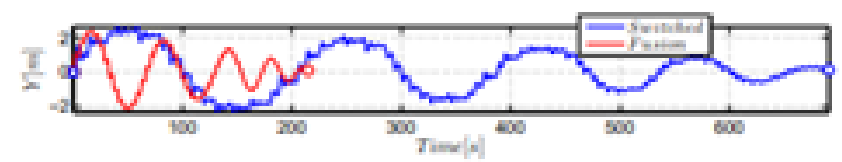

(f)

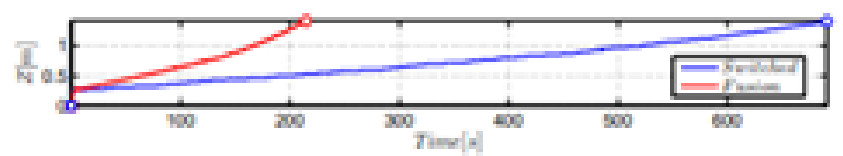

(g)

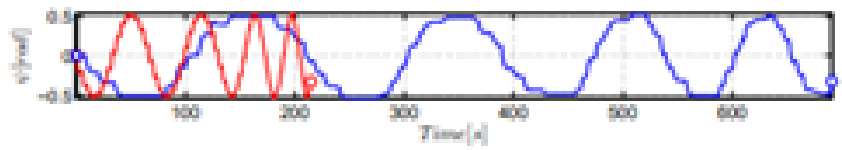

(h)

Figura 6. Grificas correspondientes al experimento 2.

\section{AGRADECIMIENTOS}

Los autores agradecen al CNPq - Conselho Nacional de Desenvolvimento Científico e Tecnológico, agencia de fomento del Ministério da Ciência, Tecnologia, Inovação e Comunicações do Brasil - y a la FAPES - Fundação de Amparo à Pesquisa e Inovação do Espírito Santo, a agencia de fomento del Estado do Espírito

Santo, Brasil -, por el financiamiento de esta investigación. Ellos también agradecen a la CAPES - Coordenacao de Aperfeicoamento de Pessoal de Nivel Superior, agencia de fomento del Ministerio da Educacao do Brasil, por la beca de estudios otorgada al doctorando Milton Cesar Paes Santos, al Instituto Federal do Espirito Santo, a la Universidade Federal do Espirito Santo y al Institute de Automatica de la Universidad Nacional de San Juan, Argentina, y CONICET, Argentina por apoyo al desarrollo de esta investigación. 


\section{REFERENCIAS}

[1] H. Eisenbeiss, "A mini unmanned aerial vehicle (uav): System overview and image acquisition," in Proceedings of the International Workshop on Processing and Visualization Using High-Resolution Imagery, Pitsanulok, Thailand, 2004.

[2] Y. Bestaoui and R. Slim, "Maneuvers for a quad-rotor autonomous helicopter," in AIAA Conference and Exhibit, Rohnert Park, California, May 7-10 2007.

[3] S. Rathinam, A. Kim, Z. Soghikian, and R. Sengupta, "Vision based following of locally linear structures using an unmanned aerial vehicle," in Proceedings of the 44th IEEE Conference on Decision and Control/2005 European Control Conference, December 2005, pp. 6085-6090.

[4] E. MacArthur, D. MacArthur, and C. Crane, "Use of cooperative unmanned air and ground vehicles for detection and disposal of mines," in Proceedings of the VI Intelligent Systems in Design and Manufacturing, vol. 5999, 2005, pp. 94-101.

[5] F. Kendoul, "Survey of advances in guidance, navigation, and control of unmanned rotorcraft systems,” Journal of Field Robotics, vol. 29, no. 2, pp. 315-378, 2012.

[6] L. Marconi and R. Naldi, "Robust nonlinear control of a miniature helicopter for aerobatic maneuvers," in Proceedings of the 32nd Rotorcraft Forum, 2006.

[7] F. Kendoul, "Optic flow-based vision system for autonomous 3d localization and control of small aerial vehicles,” Robotics and Autonomous Systems, vol. 57, pp. 591-602, 2009.

[8] T. Madani and A. Benallegue, "Control of a quadrotor mini-helicopter via full state backstepping technique," in Proceedings of the 45th IEEE Conference on Decision and Control, Dec 2006, pp. 1515-1520.

[9] D. Mellinger and V. Kumar, "Minimum snap trajectory generation and control for quadrotors," in Proccedings of the 2011 IEEE International Conference on Robotics and Automation, May 2011, pp. 2520-2525.

[10] P. Castillo, A. Dzul, and R. Lozano, "Real-time stabilization and tracking of a fourrotor mini rotorcraft," IEEE Transactions on Control Systems Technology, vol. 12, no. 4, pp. 510-516, July 2004.

[11] A. S. Brandão, M. Sarcinelli-Filho, and R. Carelli, "A nonlinear underactuated controller for 3d-trajectory tracking with a miniature helicopter," in Proceedings of the 2010 IEEE International Conference on Industrial Technology, March 2010, pp. 1421-1426.

[12] --, "High-level underactuated nonlinear control for rotorcraft machines," in 2013 IEEE International Conference on Mechatronics, Feb 2013, pp. 279-285.

[13] P. Castillo, R. Lozano, and A. Dzul, "Experimental implementation of linear and nolinear control laws,” IEEE Control System Magazine, pp. 45-55, December 2005.

[14] J. Hauser, S. Sastry, and G. Meyer, "Nonlinear control design for slightly non-minimum phase systems: Application to v/stol aircraft,” Automatica, vol. 28, pp. 665-679, 1992.

[15] A. S. Brandão, D. Gandolfo, M. Sarcinelli-Filho, and R. Carelli, "PVTOL maneuvers guided by a high-level nonlinear controller applied to a rotorcraft machine," European Journal of Control, vol. 20, no. 4, pp. 172 - 179, 2014. 
[16] A. S. Brandão, M. Sarcinelli-Filho, C. D. Rosales, and R. Carelli, "3- d positioning tasks for ruas using switched pvtol controllers,” in 2013 IEEE International Conference on Mechatronics (ICM), Feb 2013, pp. 206-212.

[17] A. G. O. Mutambara, Decentralized Estimation and Control for Multisensor Systems, 1st ed. Boca Raton, FL, USA: CRC Press, 1998, vol. 1.

[18] E. Freire, T. Bastos-Filho, M. Sarcinelli-Filho, and R. Carelli, "A new mobile robot control approach via fusion of control signals," IEEE Transactions on Systems, Man, and Cybernetics, Part B (Cybernetics), vol. 34, no. 1, pp. 419-429, Feb 2004.

[19] M. C. P. Santos, L. V. Santana, M. M. Martins, A. S. Brandão, and M. Sarcinelli-Filho, "Estimating and controlling uav position using rgbd/imu data fusion with decentralized information/kalman filter," in 2015 IEEE International Conference on Industrial Technology (ICIT), March 2015, pp. 232-239.

[20] J. Engel, J. Sturm, and D. Cremers, "Camera-based navigation of a lowcost quadrocopter," in Proceedings of the 2012 IEEE/RSJ International Conference on Intelligent Robots and Systems, October 2012, pp. 2815- 2821.

[21] L. V. Santana, M. Sarcinelli-Filho, and R. Carelli, "Estimation and control of the 3d position of a quadrotor in indoor environments," in Proceedings of the 16th International Conference on Advanced Robotics (ICAR'13), Montevideo, Uruguay, November 2013.

[22] K. Saadeddin, M. F. Abdel-Hafez, and M. A. Jarrah, "Estimating vehicle state by gps/ imu fusion with vehicle dynamics," in Proceedings of the 2013 International 